\title{
El Arribo a Liceos Públicos de Excelencia. La figura materna como recurso para el cambio en el contexto escolar
}

\author{
The arrival to Public Schools of Excellence. The maternal Figure as a \\ Resource for Change in the School context.
}

\author{
Evelyn Mejías Meneses ${ }^{1}$ \\ Alejandra Santana López ${ }^{2}$
}

\begin{abstract}
Resumen
El artículo presenta un diseño de intervención apunta a facilitar los procesos de transición de niños/as que se mueven desde escuelas básicas locales (Enseñanza Básica) a Liceos Públicos de Excelencia (Enseñanza Media) a través del fortalecimiento de la figura materna. Las madres resultan ser el principal apoyo para los niños/as y quienes ejercen activamente estrategias de involucramiento familiar en relación a los establecimientos en contextos de vulnerabilidad sociocultural.

La intervención busca fortalecer a las madres propiciando el desarrollo de habilidades en el área personal y social que faciliten su participación en los Liceos mediante estrategias de acompañamiento individual y grupal. La apuesta se centra en considerar que madres fortalecidas en su rol social en un sentido amplio generarán un impacto positivo y virtuoso de carácter ecosistémico en los niños/as, la familia y su comunidad educativa.
\end{abstract}

Palabras Clave: Intervención social, transición educativa, involucramiento familiar, madres participativas

\begin{abstract}
The article seeks presents the design of an intervention that aims to facilitate the transition processes of children from local elementary schools to public High Schools of Excellence through strengthening the maternal figure. The intervention focuses on mothers primarily due to their active support and involvement is schools in contexts of sociocultural vulnerability.

Through individual and group support strategies, the intervention seeks to strengthen mothers by promoting the development of skills that facilitate their participation in the high schools. Utilizing an ecosystem lens, the underlying premise of the program is that mothers who have developed such skills will have a positive impact on their child, family and the educational community.
\end{abstract}

Key words: Social intervention, educational transition, family involvement, participatory mothers

1. Magíster en Trabajo Social y Familia UC. Licenciada en Trabajo Social. emejias@uc.cl

2. Doctora en Ciencias de la Educación. Magister en Educación. Magíster en Psicología mención Social-Comunitaria. Licenciada en

Trabajo Social Encargada de Ética y Seguridad de la investigación UC asantana@uc.cl 


\section{Introducción}

La posibilidad de involucrar a las familias en el ámbito escolar es una demanda que las escuelas hacen en Chile y el mundo (Gubbins, 2016; Ospina-Botero, 2016; Acuña-Collado, 2016). La evidencia científica internacional ha dado cuenta de las ventajas de contar con familias involucradas en la escuela (Grolnick, 2015; Ferrara, 2015), concentrándose los estudios en educación inicial y primer ciclo de educación básica. A nivel nacional se han desarrollado investigaciones en esta línea centradas en la etapa de educación básica (Reininger y Santana, 2017; Gubbins y Otero, 2018; Mejías, Santana, Saracostti, Reininger y Lara, 2019), ellas han destacado la importancia de disponer de estrategias de involucramiento familiar diferenciadas cuando se trata de niños/as y jóvenes de enseñanza media, especialmente cuando transitan desde escuelas locales (de sus barrios) a Liceos Públicos de Excelencia ${ }^{3}$, parte de los resultados de estas investigaciones destacan el rol de madres (u otras figuras maternas), dada la incidencia en el potencial desarrollo de los niños/as (Arancibia, $1997^{4}$; Jimenez, Ito y Macotela, 2010; Grolnick, 2015; Mercado y Montaño, 2015).

En respuesta a estos hallazgos locales (Mejías, 2014; Reininger y Santana, 2017; Santana y Reininger, 2017; Mejías, Santana, Saracostti, Reininger y Lara, 2019) se opta por desarrollar un diseño de intervención social factible de implementar en establecimientos de Educación Media apuntando a facilitar los procesos de tránsito desde la Educación Básica a través del fortalecimiento de las madres en su rol parental y social más amplio apostando por su positiva incidencia en los procesos de aprendizajes de los niños/as, en la familia y la comunidad. Este diseño puede ser usado como un piloto factible de implementar en establecimientos de educación media que estén recibiendo estudiantes desde la educación básica. Si bien, la idea se basa en la experiencia de una implementación experimental que se enfocó en el tránsito a Liceos Públicos de excelencia (Mejías, Santana, Saracostti, Reininger y Lara, 2019), se puede replicar en contextos similares.

La propuesta que se presenta se gesta para apoyar los procesos de tránsito de Educación Básica a Educación Media, pues estos revisten particulares complejidades, se identifican como un momento de cambio crítico debido a las diferencias en la organización curricular, el tiempo, el espacio y las relaciones sociales entre estos dos espacios (Filardo, 2016; Bernardi y Cebolla, 2014; Ames y Rojas, 2011). En la mayoría de los escenarios, la transición de primaria a secundaria implica una transferencia física de una escuela a la otra, además de la simultaneidad de estos cambios con el comienzo de la pubertad y la adolescencia - que en algunos casos significa también el comienzo de la vida adulta - convierte a este proceso en uno particularmente intenso (Ames y Rojas, 2011). En el contexto chileno el tránsito de una escuela local a un liceo público de excelencia implica para los niños/as y sus familias múltiples exigencias, al tratarse de un sistema altamente selectivo y centrado principalmente en el logro académico, por lo tanto, se justifican ampliamente las estrategias que den soporte a este tránsito y que consideren las dimensiones familiares y escolares en juego.

El artículo se estructura en tres secciones. La primera introduce al lector en la temática y aporta las principales aristas de problematización respecto a las transiciones educativas, las ventajas del involucramiento familiar y la relevancia del rol materno en los procesos de aprendizaje de los niños/as. Incorporando los principales referentes contextuales y teóricos-conceptuales, profundizando en aspectos propios de la política educativa, la renovada política de participación familiar chilena y conceptualizaciones asociadas al involucramiento familiar colaborativo.

La segunda sección muestra el diseño de la intervención propiamente tal, destacando los supuestos de intervención, los objetivos y los referentes teóricos a la base. Desde el punto de vista operativo, se exponen los modelos de intervención y su articulación a través de las estrategias específicas de intervención, en este caso, expresadas en el trabajo individual y colectivo con las madres de los niños/as y jóvenes.

3. El sistema escolar ha contado con un conjunto de Liceos Públicos de Excelencia (LPE) los cuales, además de lograr un alto desempeño académico entre sus estudiantes, se caracterizan por su alta selectividad en el ingreso de sus estudiantes

4. El estudio de Arancibia (1997) fue uno de los pioneros a nivel nacional en destacar la capacidad de agenciamiento de las madres. A partir de esto, se fue incorporando también en la política educativa el rol y perfil materno como un marcador de tendencia de éxito escolar. 
Finalmente, en la tercera sección se desarrolla una reflexión respecto al horizonte de cambio al que apunta esta intervención, las condiciones mínimas y necesarias para poder implementar la propuesta en establecimientos y las proyecciones que tiene un trabajo de esta naturaleza.

\section{Relación familia-escuela: el involucramiento familiar como recurso de intervención para la transición escolar}

El diseño de la propuesta de intervención "Transición a la Enseñanza Media. El arribo a Liceos Públicos de Excelencia" se sustenta en una serie de antecedentes empíricos y contextuales que evidencian un vacío de conocimientos y de propuestas de intervención que involucren a las familias en situaciones coyunturales como el tránsito a la enseñanza media y las particulares demandas de involucramiento en esta etapa del ciclo vital de familias en condición de desventaja sociocultural.

La fundamentación del diseño de intervención "Transición a la Enseñanza Media. El arribo a Liceos Públicos de Excelencia" se sustenta en los principales referentes de la literatura nacional e internacional especialmente aquellos derivados del School Social Work americano, área que ha aportado los principales sustentos del trabajo social escolar desde la perspectiva anglosajona (Kelly, Thompson, Frey, Klemp, Alvarez y Cosner, 2015; Demissie y Brener, 2017). Adicionalmente, se consideró a nivel local el resultado de una investigación cualitativa que recogió las experiencias de vida de madres que han sido agentes de acompañamiento clave para niños/as que han realizado transición de escuelas locales a Liceos Públicos de Excelencia en Chile (Mejías, 2014) 5 .

En la actualidad, desde diversas intervenciones sociales estudiadas en el ámbito escolar se busca involucrar a las familias en los procesos de aprendizaje y mejorar la relación con la escuela favoreciendo una alianza virtuosa entre ambas esferas (Gubbins, 2012; Romagnoli y Cortese, 2015; Ferrara, 2015; Gubbins e Ibarra, 2016; Reininger y Santana, 2017; Santana y Reininger, 2017). Sin embargo, esta constatación no ha logrado iluminar qué integrantes de la familia son quienes mayormente están presentes en el compromiso escolar y qué particularidades tiene este compromiso en la etapa de transición de enseñanza básica a enseñanza media, pues la mayoría de los antecedentes reportados por la literatura se centra en el primer ciclo básico escolar (Reininger y Santana, 2017, Mejías, Santana, Saracostti, Reininger y Lara, 2019).

La influencia de la familia en el desarrollo de los niños/as y especialmente en el escolar posee múltiples explicaciones, esta apuesta de intervención se sustenta en diversos enfoques que avalan la influencia de la familia en los procesos de aprendizaje escolar, ya sea desde vertientes sociológicas y/o culturalistas (Dabas, 2005; Lareau y López, 2012, Lareau, 2015) o enfoques de mejora escolar (Elmore, 2010; Anderson y Carrasco, 2013), pues se comprende que la familia explicaría parte importante de los aprendizajes de los niños/os y de sus logros académicos y no académicos (Grolnick, 2015; Ferrara, 2015).

En Estados Unidos se reconoce un grupo especializado de intervenciones que apuntan directamente a incidir en la relación entre la familia y la escuela, denominadas involucramiento parental, referido a la disposición familiar hacia la escuela y los aprendizajes de los niños, considera las prácticas conductuales y verbales en el hogar, así como las que se despliegan en las actividades que organiza la escuela (Duppong, Lambert, January y Huscroft, 2017; Park y Holloway, 2017). Se concibe como un proceso asociativo entre la escuela y la familia su abordaje tiene una data de más de 30 años en ese país, mutando desde aproximaciones

5. El trabajo completo de la autora principal correspondió a su tesis de magister en Trabajo Social y Familia (Escuela de Trabajo Social PUC) que consideró una dimensión de investigación y una dimensión de intervención. El énfasis de este artículo estará en exponer el diseño de intervención fruto de este trabajo global. El desarrollo de la dimensión investigativa de este trabajo se encuentra publicada en (Mejías, Santana, Saracostti, Reininger y Lara, 2019). 
más tradicionales, verticales y rígidas de la relación familia - escuela a perspectivas colaborativas vigentes en la actualidad (Deslandes, 2019; Yi Hung y Power, 2018; Epstein, 2018; Reininger y Santana, 2017). Estas disposiciones de orientación colaborativa resultan útiles para plantear de qué manera las madres y su entorno pueden incidir positivamente en las transiciones escolares.

El interés por centrarse en intervenciones que abordan el vínculo con las familias surge de los resultados de investigaciones que muestran una correlación positiva entre el involucramiento parental y el rendimiento académico (Grolnick, 2015; Ferrara, 2015), la disminución en tasas de deserción escolar (Reinke, Smith y Herman, 2019); el impacto positivo en el desarrollo social y emocional de los niños/as, expresándose en una mayor capacidad de autorregulación y mayores niveles de desarrollo de las habilidades sociales (Roy y Giraldo-García, 2018).

Las intervenciones escolares en que se involucra a las familias en contextos de desventaja operan como dispositivos para abordar situaciones familiares que se superponen a la dimensión educativa de los niños/as: la ausencia de los padres ${ }^{6}$ como actores fundamentales en la formación de sus hijos/as, la percepción de los padres de no tener un rol que cumplir en materia educativa, dificultades familiares diversas que influyen en la condición de salud biopsicosocial de los niños, recursos socioculturales escasos y/o ausencia de conciencia de la relevancia de la educación para sus hijos (Goldfard, Locher, Preskitt, Becker, Davies y Sen, 2017; Coe, Davies y Sturge-Apple, 2017; Vásquez, Zuluaga y Fernández, 2017).

\section{Involucramiento familiar colaborativo}

El diseño de esta intervención recoge elementos del involucramiento familiar colaborativo, considerando incorporar a las madres en los procesos de transición escolar. Este tipo de involucramiento surge como alternativa a la vinculación que tradicionalmente se ha dado entre familia-escuela, caracterizada por una relación vertical rígida, con énfasis normativo y directivo de parte de la escuela, marcada por interacciones tensas, de poca colaboración y con pautas de involucramiento jerárquicas desde la escuela. Con frecuencia el sistema escolar se ha vinculado con la familia para señalarles las dificultades, los problemas y las deficiencias que los niños pueden tener en la sala de clases ya sean estas cognitivas, emocionales, sociales o conductuales; perdiendo la oportunidad de trabajar con las familias como aliados que trabajan por un meta común: el aprendizaje y desarrollo de los niños/as.

Así, es posible reconocer que cuando se logra implementar un involucramiento familiar colaborativo en el contexto escolar, la escuela sin duda pasa a ser un factor protector para los niños y sus familias (Epstein, 2018). Para esto es útil estimar y medir este involucramiento en las trayectorias educativas de los niños/as; hacerlo, permite fortalecer áreas, redirigir acciones e innovar en mejoras a este respecto. Tener estos datos a la mano es una herramienta clave para la gestión de la escuela en materia de involucramiento permitiendo tomar decisiones de manera fundamentada.

Las manifestaciones del involucramiento familiar colaborativo son variadas, incluyen actividades en el hogar, apoyo a las actividades escolares y en general la disposición de la familia a participar en la esfera escolar. Esto puede incluir: apoyando las tareas escolares, asistiendo a actividades convocadas desde la escuela, participando en clases o capacitaciones dirigidas a los padres, apoyando la labor pedagógica en aula, comprometiéndose con cambios en la escuela en favor del bienestar de los niños o participando en la toma de decisiones de aspectos relativos a la escuela (Reininger y Santana, 2017).

6. Para efectos de este artículo los padres o quienes ejercen el rol parental no se limita a los vínculos biológicos, sino que se extiende a quienes ejercen la labor de cuidado, protección, socialización y entrega de afectos a los niños/as, pudiendo ser realizado por otros familiares o adultos significativos. Lo que es consistente con las características socioculturales de los contextos de desventaja nacional. 
Es posible reconocer que existen elementos que comúnmente se encuentran en interacción con el tipo de involucramiento familiar desplegado, tales como los recursos o capital social y cultural de la familia y el entorno, la disposición a confiar en la escuela y en el profesor jefe, la disposición familiar hacia los aprendizajes de sus niños y niñas, las experiencias previas y personales de los apoderados con su propio proceso de escolarización y los intereses de participación en las actividades de la escuela por parte de las familias (Reininger y Santana, 2017).

\section{La particular influencia de la figura materna en procesos de involucramiento familiar en procesos de transición escolar}

En el caso del diseño de intervención "Transición a la Enseñanza Media. El arribo a Liceos Públicos de Excelencia" se contemplan condiciones que favorecen el proceso de cambio, caracterizado por el acompañamiento materno a los niños/as durante la educación, el apoyo y disposición para re-editar las formas de soporte en la transición a la enseñanza media en el contexto de Liceos Públicos de Excelencia, escenarios escolares caracterizados por mayores exigencias académicas para los niños/as y escasos mecanismos de involucramiento para la familia (Mejías, 2014; Mejías, Santana, Saracostti, Reininger y Lara, 2019). Es decir, se trata de familias que han desarrollado ciertos capitales culturales, que se traducen en recursos, expectativas y prácticas que favorecen el involucramiento familiar (Chacón, Chacón, Alcedo y Suárez, 2015).

Se encuentra amplia evidencia de la relación entre la influencia parental y la toma de decisiones juveniles relativas continuar estudios terciarios - sin hacer distinción entre figura materna y paterna - indican que "mostrar interés y proporcionar apoyo a los planes académicos facilita el desarrollo de la carrera. Entre las acciones concretas destacan: el modelado de comportamientos, la protección, la transmisión de adecuadas expectativas de éxito, la comprensión, la provisión de experiencias de aprendizaje o iniciar o facilitar el contacto con personas o instituciones que ayuden a la comprensión de las diversas rutas educativas y profesionales" (Fernández, García y Rodríguez, 2016). En el caso de los niños/as que transitan de educación básica a media y de escuelas locales a liceos públicos de excelencia se reconoce la relevancia de este tipo de acciones por parte de las madres particularmente (Mejías, 2014; Mejías, Santana, Saracostti, Reininger y Lara, 2019).

La propuesta de intervención "Transición a la Enseñanza Media. El arribo a Liceos Públicos de Excelencia" hace una opción por el trabajo con las madres (o figuras maternas), aun cuando esta aproximación se reconoce desde un enfoque familiar (Aylwin y Solar, 2002), se sustenta en fortalecer habilidades y competencias maternas apostando por facilitar y propiciar una sana transición a la enseñanza media en los niños/as. Se destaca que las madres son más activas participando en los procesos educativos de los niños/as y serían las que mantienen mayor contacto con los establecimientos (Pérez, Álvarez y Cubillos, 2016). Se postula que: "las madres son las personas más importantes en las relaciones familia-escuela. Entre el 70-80\% de la relación familia-escuela es llevada a cabo por las madres de los niños/as. Ellas ayudan a sus hijos/as con sus deberes y participan en la escuela en encuentros y actividades" (Jensen, Joseng y Lera, 2007, p. 5).

El trabajo pionero de Arancibia (1997) fue una fuente de motivación importante para generar este diseño, su investigación alude al carácter de habilitadoras de algunas madres en las trayectorias formativas de los niños/as, estas realizan acciones en su vida cotidiana que permiten que sus hijos/as lleguen con mejores posibilidades de desarrollo cognitivo e intelectual a la situación educativa, y por tanto con más posibilidades de lograr un buen rendimiento, no repetir de curso, no desertar de la escuela y alcanzar altos niveles de escolaridad, y repetir este círculo virtuoso en el futuro. Estas madres dan prioridad a la educación ante las restantes necesidades y la visualizan como una vía de movilidad social. En síntesis, las características de estas madres apelan a: niveles más altos de escolaridad que su promedio, mayores expectativas de rendimiento 
respecto de los hijos/as, y presentan conductas efectivas para apoyar la educación de sus hijos/as. Estos hallazgos fueron nuevamente encontrados en investigaciones en el contexto nacional (Mejías, 2014; Mejías, Santana, Saracostti, Reininger y Lara, 2019), aportando fundamentos a este diseño de intervención.

Estudios internacionales también se han detenido en el rol materno y su influencia en los aprendizajes de los niños/as. Para Jiménez, Ito y Macotela (2010) las condiciones del hogar y las creencias y prácticas de las madres que enfatizan y estimulan el aprendizaje escolar, favorecen la motivación de sus hijos/as hacia el aprendizaje. Se destacan particularmente actitudes como la confianza en las capacidades de su hijo/a y el interés y entusiasmo con que realiza su labor de apoyo. En un estudio con madres europeo-americanas se analizó el impacto de sus motivaciones en el involucramiento y rendimiento escolar de sus hijos/as, descubriendo que cuando las madres están más comprometidas con las actividades escolares, se sienten más autónomas y disfrutan más del involucramiento, sintiéndose más seguras y menos estresadas. Además, se apreció una internalización de las motivaciones maternas en los niños/as, impactando en las competencias académicas, niveles de lectura y autoestima (Grolnick, 2015).

En investigaciones nacionales (Mejías, 2014; Mejías, Santana, Saracostti, Reininger y Lara, 2019), se aprecia que la transición de los niños/as desde las escuelas locales a los Liceos Públicos de Excelencia, implicó un impacto en ellos y en sus familias, pues se trata de establecimientos masivos, con nuevas dinámicas de relaciones entre los docentes y los apoderados, con nuevas y altas exigencias para los estudiantes, con heterogeneidad de alumnos y apoderados desde el punto de vista sociocultural, condiciones que implican un proceso de adaptación a una nueva forma de posicionarse como estudiantes y como apoderados (Santana, 2010a; Santana, 2010b). Además, también hay que considerar que, en el caso de los niños/as, estos/as se encuentran en una etapa vital marcada por cambios personales y psicológicos propios de la pubertad y adolescencia, lo que también puede implicar cambios en la relación con sus padres o cuidadores, en los límites de la relación que establezcan con ellos y por ende, implica que también se produce un proceso de adaptación en la relación que los adultos pueden establecer con los propios hijos/as y en las posibilidades de participar en sus actividades escolares y/o personales. Destaca que “... se percibe más fácil participar en la educación básica que en la enseñanza media, pues en la primera existen mayores necesidades de atención de los padres por parte de los niños, mientras que en la educación media se requiere de competencias parentales más complejas para tratar con los adolescentes" (Sánchez, Reyes y Villarroel, 2016, p. 350).

Mejías (2014) exploró respecto a cómo vivencian las madres su experiencia inicial de participación en los liceos de excelencia a los que han ingresado recientemente sus hijos/as. Ellas reportan un cambio en el tipo de apoyo que es requerido, así en las escuelas básicas locales, tenían una participación más activa apoyando las tareas y diversas demandas escolares; en el contexto de los liceos de excelencia, se presenta un escenario de incertidumbre, donde se aprecian dificultades para participar, expresadas en: pocas instancias promovidas desde los liceos, percepción de distinciones de clases sociales entre apoderados, autopercepción de ineficacia en el apoyo que puedan dar a sus hijos/as, falta de información desde la escuela, entre otras. Estas dificultades podrían explicarse desde las características de estos establecimientos, tales como: focalizados en el rendimiento académico, con escasos espacios de acogida a la familia, énfasis individualista de los logros, enfocado en la autonomía de los estudiantes.

Las instancias de participación de las madres en los establecimientos de excelencia se caracterizan por desarrollarse a un nivel incipiente asociándose a actividades como: respuesta a las obligaciones mínimas escolares, apoyo en tareas, mantención de la comunicación con el establecimiento, obtención de información sobre el proceso de los hijos/as; realización de acciones voluntarias; asistencia a determinadas actividades de los hijos/as, entre otras (Mejías, 2014). 
De esta manera, este diseño de intervención se fundamenta en: la relevancia de la relación familia-escuela, la particular influencia de las madres en los procesos de aprendizaje de los niños/as y los hallazgos locales respecto a la experiencia inicial de madres que han acompañado los procesos de transición académica de niños/as que han pasado de escuelas locales a liceos de excelencia en contextos de desventaja sociocultural. Haciendo una opción para potenciar el rol materno a través del fortalecimiento de habilidades y competencia personales que faciliten este ejercicio en nuevos escenarios formativos de sus hijos/as.

\section{La política educativa: oportunidades para el desarrollo de involucramiento familiar en procesos de transición escolar}

En relación a los antecedentes contextuales es relevante destacar la actual política educativa en el contexto nacional, en la que se evidencia un reconocimiento a la participación de las familias en las comunidades escolares apuntando a una comprensión más integral del fenómeno educativo, lo que se expresa en la actual Ley General de Educación (2009) y los múltiples cuerpos legales y programáticos que apuntan a un abordaje activo de las condiciones de aprendizajes de los niños/as, validando a las comunidades y a las familias. Particular mención tiene la renovada Política de Participación de Fa familia y la Comunidad (MINEDUC, 2017).

La Política de Participación de la Familia y la Comunidad en instituciones educativas (2017), se reformuló luego de un trabajo diagnóstico y reflexivo de diversos actores sociales. Su énfasis está en evidenciar el aporte de la familia y la comunidad al fortalecimiento del sistema educativo potenciando la integración de la escuela con su entorno y la generación de espacios para la ciudadanía, convivencia y participación. Su objetivo general es "aportar al desarrollo integral del estudiantado y a mejorar la calidad educativa, a través de la generación de condiciones para la participación e involucramiento de las familias y la comunidad en las instituciones educativas" (MINEDUC, 2017, p. 31). Esta política canaliza su accionar en tres líneas estratégicas: diálogo y colaboración entre actores educativos, apoyo a la formación de actores educativos para la participación e integración de la escuela a la comunidad local.

Por su parte, al buscar alternativas que permitan dar cabida a intervenciones de carácter familiar en la escuela y que pueden ser financiadas, surge como oportunidad los recursos provenientes de la Ley SEP (2008). Esta ley tiene como propósitos: ayudar a corregir la inequidad histórica del sistema actual en lo que a calidad y oportunidades refiere; relevar que la calidad de vida de las personas se define en las oportunidades educativas; hacerse cargo desde el nivel preescolar a la enseñanza media (este último nivel es incorporado el año 2011); otorgar un incentivo al sostenedor interesado; focalizar los esfuerzos en aspectos educacionales; y abordar un aspecto clave para dar mayor justicia, protección e igualdad a los sectores más vulnerables y alejados territorialmente (Muñoz, Marfán, Pascual, Sánchez, Torres y Von Hausen, 2010). Las escuelas utilizan los recursos de la SEP a través del financiamiento a planes de mejoramiento, lo que puede significar, desde la contratación de profesionales del área social hasta la implementación de estrategias de apoyo familiar que requieran un abordaje interdisciplinario en la escuela.

A partir de estos marcos legislativos que regulan el funcionamiento escolar y particularmente operan como condiciones favorables para el desarrollo de estrategias de intervención con las familias, como lo es el diseño propuesto en este artículo.

7. Maureira (2016) desarrolla una síntesis de los principales hitos legislativos que el MINEDUC ha impulsado para abordar los problemas de equidad y calidad en el sistema escolar chileno. 


\section{Diseño de intervención: Modelo de Intervención "Transición a la Enseñanza Media. El arribo a Liceos Públicos de Excelencia"}

La propuesta de intervención se enfoca en el desarrollo y fortalecimiento de procesos individuales y grupales con las madres para propiciar el desarrollo de diferentes competencias, capacidades, habilidades en cada una de ellas, las que serán útiles tanto para desenvolverse en el ejercicio de su participación en los establecimientos educacionales y en otras áreas de su interés, sirviéndose para optimizar su participación en distintos otros espacios sociales.

En primer lugar, la intervención busca fortalecer a las madres para que puedan enfrentar el proceso de inserción de sus hijos/as a los establecimientos educacionales de excelencia académica, considerando un proceso de acompañamiento donde tengan instancias en las que puedan abordar las dificultades o las inquietudes que enfrenten e incluso puedan incorporar conocimientos nuevos que les faciliten su rol.

En segundo lugar, con la intervención propuesta, se apunta a que las madres se potencien, trascendiendo su rol materno, logrando un proceso de desarrollo integral, en el cual desplieguen habilidades o capacidades que las fortalezcan para desenvolverse en distintos espacios de interacción social.

En tercer lugar, se pretende que las madres, a través de un proceso grupal, puedan compartir su experiencia con otras mujeres que se encuentran viviendo procesos similares, configurado espacios de contención, colaboración y aprendizaje. Adicionalmente, se busca que las madres logren la conformación de una red de apoyo para sí mismas, que podría contribuir para enfrentar los desafíos del cambio y facilitar la adaptación a este.

La apuesta por las mujeres que ejercen el rol parental-materno responde a los antecedentes presentados en las secciones anteriores de este artículo, los que en síntesis se refieren a: 1 . Reconocer que son las madres las que asumen mayor protagonismo en el proceso educativo de los hijos/as; 2. Identificar a madres con preocupación por mejorar la calidad de educación de sus hijos/as, las llamadas "madres habilitadoras"; y 3. Se releva que las madres de escasos recursos presentan dificultades para ejercer su participación en el proceso educacional de sus hijos/as en colegios de excelencia académica.

\section{Destinatarios de la propuesta}

Los destinatarios directos del diseño son mujeres que ejercen el rol parental-materno pertenecientes a segmentos socioculturales desaventajados a cargo del cuidado de niños/as que se encuentren en el tránsito a la enseñanza media insertándose en Liceos Públicos de Excelencia (o establecimientos de similares características).

Los destinatarios indirectos de la intervención, serían los hijos o hijas de estas madres, otros integrantes del grupo familiar y otros actores participantes del proceso educacional (comunidad educativa de los establecimientos educacionales).

\section{Enfoques teóricos que sustentan el diseño de intervención}

La intervención se sustentará en tres perspectivas (ver gráfica 1): sistémica, ecológica y de las fuerzas.

La perspectiva sistémica es considerada como referente en cuanto al enfoque de comprensión de la familia y sus dinámicas. "Un sistema se puede entender como una totalidad en la cual sus partes, en nuestro caso los integrantes de la familia, son interdependientes entre sí, se influyen mutuamente, lo que supone que lo que le suceda a alguno de sus miembros influye a los demás y a la familia como un todo" (Aylwin y Solar, 2002, p. 107). En este caso, el cumplimiento del rol parental-materno conlleva a la interrelación de la familia con diversos sistemas necesarios para el bienestar del grupo (Chinchilla, 2015). Uno de ellos es la interacción del sistema familiar con el sistema educacional, orientándose ambos a la formación y desarrollo cognitivo y sociocultural de los jóvenes (Gomila y Pascual, 2015). 
De manera complementaria, la perspectiva ecológica en el marco de esta intervención aporta elementos analíticos asociados a la relación entre las personas y sus ambientes físicos y sociales (Greenberg, Domitrovich y Brumbarger, 2002; Greene, 2008). Desde esta perspectiva "la ecología se ocupa de ese sensible balance que existe entre los seres vivos y sus ambientes y de las maneras en que ese balance puede ser mantenido y mejorado" (Hartman y Laird, 1983 en Aylwin y Solar, 2002, p. 124). Es importante considerar a las madres desde las características propias del contexto social en el cual se desenvuelven y la intervención propuesta pretende fortalecerlas para que logren desplegar habilidades que les faciliten la interacción con dicho entorno y más aún, con nuevos entornos. De esta manera, madres fortalecidas en su rol propiciarán intercambios sanos con los ambientes relativos a lo escolar - en este caso establecimientos nuevos y de alta exigencia para los jóvenes - y a lo familiar, incluso fortaleciéndose el vínculo parental-materno, logrando interactuar mejor en dichos entornos.

La propuesta de intervención también considera en sus referentes teóricos la perspectiva de las fuerzas aportando recursos comprensivos para aproximarse a las madres desde su rol social de mujer, no solo limitado a la dimensión parental-materno. La propuesta de intervención concibe a las madres como personas que cuentan con recursos propios y múltiples potencialidades que el proyecto busca aportar a su desarrollo.

La perspectiva de las fuerzas propone supuestos que se vuelven relevantes para la intervención, porque intencionan reconocer a las madres desde sus recursos y no desde sus carencias o vulnerabilidades. Entre estos supuestos se destacan: "todas las personas, familias y los ambientes cuentan con fuerzas que pueden ser activadas para mejorar la calidad de vida; la motivación de las personas y familias aumentan o se fomentan con un énfasis consistente en las fuerzas tal como ellas las definen; todos los ambientes hasta los más carenciados tienen recursos (De Jongh y Miller, 1985 en Aylwin y Solar, 2002, p. 194).

\section{Objetivos}

El objetivo general del diseño de intervención apunta a: Contribuir a que madres de sectores vulnerables con hijos o hijas que asisten a establecimientos educacionales de excelencia académica desarrollen habilidades en el área personal y social que les faciliten la participación en los liceos, a través de estrategias de acompañamiento individual y talleres grupales.

Los objetivos específicos de la intervención son:

1. Reconocer la importancia de la participación de la familia en la escuela, para el pleno desarrollo educacional de sus hijos o hijas, a través de un proceso de acompañamiento a madres de sectores vulnerables.

2. Fortalecer la autoestima y habilidades sociales de las madres de sectores vulnerables con hijos o hijas que asisten a establecimientos educacionales de excelencia académica, a través de instancias de acompañamiento y talleres, a fin de que visibilicen sus capacidades para participar en el proceso educacional de sus hijos/as.

3. Apoyar el desarrollo de habilidades comunicativas en las madres a través de orientación y difusión de información relevante, a fin de mejorar el vínculo con los establecimientos educacionales.

4. Fortalecer la autoayuda y las redes de apoyo sociales en las madres a través de su participación en talleres grupales. 


\section{Metodología de la intervención}

\section{Estrategias de intervención}

La propuesta de intervención contempla dos estrategias de trabajo con las madres: trabajo individual y trabajo colectivo-grupal.

Sesiones individuales: Esta estrategia consiste en el desarrollo de entrevistas semiestructuradas dirigidas a las madres por parte de los profesionales a cargo de la intervención, se contará con un guion, de carácter flexible que dé cabida a incorporar otras áreas de indagación (Taylor y Bogdan, 2002) o de respuesta a las inquietudes de las madres. En estas instancias se profundizará en los temas que emerjan de las sesiones grupales y/o los temas emergentes que llevan las madres. Se trata de un espacio de clarificación de dudas y también tendrá un carácter informativo y orientador sobre consultas que tengan las madres respecto del proceso educativo de sus hijos/as. También se contempla el establecimiento de tareas que se chequearán en las sesiones siguientes enfocadas a fortalecer el proceso de desarrollo de las madres. Se estiman entrevistas mensuales para cada participante.

Sesiones colectivo-grupales: Esta estrategia es canalizada a través de las sesiones de talleres en las que participarán las madres. Los talleres abordarán temáticas como: alianza familia-escuela, autoestima, comunicación, resolución de conflictos y redes de apoyo. Se estima desarrollar las sesiones dos veces al mes.

Las sesiones de talleres se estructurarán en cuatro momentos:

a) Revisión de contenidos de la sesión anterior - desarrollo: Tiene por objetivo recordar lo que se trabajó en la sesión anterior del taller, generando la instancia para clarificar posibles dudas y para que las participantes compartan las reflexiones que pudieron realizar en torno a la sesión. También en este momento se consultará por posibles situaciones que hayan vivido en el tiempo transcurrido entre las sesiones y que tengan relación con los temas abordados. Además, se describirá brevemente el tema y las actividades que se realizarán ese día. En este momento, se podrán desarrollar algunas actividades o dinámicas.

b) Momento de pausa: Instancia que busca que las participantes compartan y dialoguen entre ellas en un contexto más informal y relajado con el fin de favorecer sus vínculos y constituirse en una red de apoyo para sí mismas. Los profesionales también participan de este momento como una forma de generar confianza y aproximarse a la dinámica del grupo.

c) Compartiendo conocimientos: En este momento se realizará la exposición del tema específico planificado para la sesión. Esto se llevará a cabo tanto de forma expositiva como a través de dinámicas grupales o actividades de reflexión.

d) Cierre y evaluación: Se realizará el cierre de cada sesión, construyendo una síntesis de lo trabajado, recordando la fecha de la próxima sesión y motivando a las participantes a continuar asistiendo al grupo. También, de acuerdo con el diseño de la sesión, en este momento se realizaría una breve evaluación de la misma.

\section{Momentos de la intervención}

Al ubicar la propuesta temporalmente, es posible identificar cinco momentos. Estos no se desarrollan en forma lineal necesariamente pues pueden superponerse y ajustarse. La extensión de cada etapa también puede variar conforme a las particularidades del contexto privilegiando la flexibilidad en la implementación. El gráfico 1 muestra los principales momentos, articulando con las estrategias de intervención contempladas. 


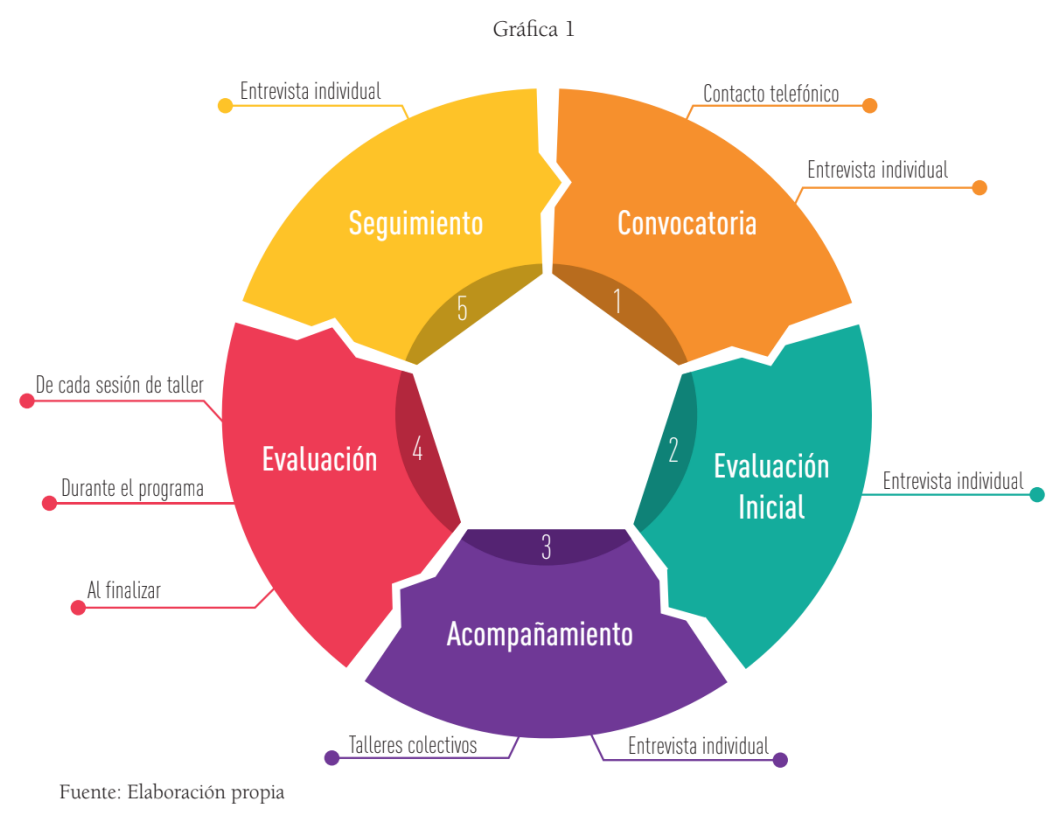

Las principales actividades asociadas a cada momento son:

Convocatoria de las madres: Contacto telefónico con las madres para acordar una primera entrevista. En esta instancia se explicará brevemente en qué consiste la intervención y se les invitará a participar. Se espera que en este espacio confirmen su participación. En caso de aceptar, firmarán un compromiso de participación y un acuerdo de confidencialidad para las instancias grupales en que se aborde información personal y/o sensible de cada una.

Diagnóstico inicial: Desarrollo de la primera entrevista semiestructurada individual con cada madre. El objetivo de esta instancia es conocer la percepción respecto del proceso del cambio de establecimiento y nivel educativo que enfrentarán con sus hijos/as. Se indagan sus inquietudes, expectativas y otros temas emergentes. La información recopilada en las entrevistas es un insumo para el diseño de las temáticas de los talleres. El equipo profesional analiza el contenido de las entrevistas, agrupa las respuestas por categorías y se van considerando en las temáticas y profundidad de estas en los talleres.

Acompañamiento: Esta etapa combina las sesiones de entrevistas semiestructuradas individuales y sesiones de taller. Es en las entrevistas con las madres donde se abordan las inquietudes personales y se va acompañando su proceso individual respecto a los avances y/o dificultades que se van identificando con el transcurso de la intervención. En paralelo, se realizan sesiones de talleres grupales en los cuales se abordan las temáticas relacionadas con los objetivos específicos de la intervención y tomando en cuenta temáticas que emergieron en el Diagnóstico Inicial.

Evaluación:La evaluación está presente transversalmente en los distintos momentos del diseño, considerándose evaluaciones de procesos y de logro de los objetivos propuestos. Para la evaluación del proceso se contempla analizar algunas sesiones de taller, seleccionadas al azar, identificando aspectos de funcionamiento que no se estén logrando, la pertinencia de las temáticas y metodologías usadas, la participación de las madres, entre otros aspectos. En cuanto a la evaluación de logros, está se orienta por una matriz de objetivos, metas e indicadores que permiten estimar el tipo y nivel de logro, cualitativa y cuantitativamente.

Seguimiento: Se realizará una vez que la intervención haya concluido, aproximadamente tres meses después. Se contempla realizar una entrevista semiestructurada individual mensual con cada una de las madres, apuntando a monitorear su actual experiencia de participación en el Liceo de sus hijos/as. Esta instancia también se usará como posibilidad para reforzar los temas trabajados en las estrategias individuales y grupales.

Las herramientas metodológicas que propone utilizar este diseño se sustentan en los enfoques teóricos anteriormente descritos y se enmarcan en modelos de intervención, es decir en constructos que van aportando a la coherencia de los recursos técnicos elegidos para implementar la intervención. 
Para esto se enfocó la búsqueda en modelos de intervención que permitieran alcanzar los objetivos propuestos, definiéndose tres de ellos: el modelo centrado en la relación de la familia con su medio; los modelos de intervención psicoeducativa y de intervención grupal.

El modelo de intervención centrado en la relación de la familia con su medio, se refiere a la intervención orientada en las relaciones de la familia con otros sistemas sociales (Aylwin y Solar, 2002; Liang y Zhang, 2016). En este caso, se abordarán temas vinculados a la relación de la familia con el sistema educativo. Esta modalidad de intervención, considera "(...) la construcción de una mayor competencia en las familias, en otorgarles un mayor poder, y en ayudar a las personas a manejar efectivamente sus vidas y sus relaciones con su medio ambiente" (Aylwin y Solar, 2002, p. 107). Este modelo será utilizado transversalmente en la implementación, es decir, es posible reconocer dimensiones del modelo en las diferentes fases de la intervención y en las actividades individuales y colectivas. Se evidenciará en el trabajo individual - en las sesiones de entrevistas individuales - a través de la intervención directa y el acompañamiento del proceso que lleve a cabo cada madre, como en el trabajo grupal correspondiente a las sesiones de talleres.

Por su parte, el modelo de intervención psicoeducativa se orienta hacia la adquisición y fortalecimiento de capacidades desde los recursos de los propios involucrados, lo que resulta coherente con los objetivos que se plantea la intervención (Montiel-Castillo y Guerra-Morales, 2016; Vélaz, López, Expósito y González, 2016). Este modelo aporta: "su carácter formativo y educativo. Se trata de ofrecer a los padres oportunidades de aprendizaje y desarrollo; suponen una acción formativa que pretende optimizar el desempeño parental mediante el fortalecimiento de competencias y no desde planteamientos más tradicionales de superación de deficiencias (...) busca proporcionar a los padres y las madres los conocimientos y las estrategias necesarias para la adquisición de habilidades educativas pero, al mismo tiempo, tratan de promover en estos progenitores la seguridad y la satisfacción con el rol parental, ambas necesarias para ampliar las habilidades relacionadas con su desarrollo adulto y lo que se ha denominado la agencia personal" (Hidalgo, Menéndez, Sánchez, Lorence y Jiménez, 2009, p. 418).

En tercer lugar, se utilizará el modelo de intervención grupal que será aplicado a las sesiones de talleres (Imbernon y Medina, 2008, Landaverde, 2011). Esta instancia busca que las madres se conozcan entre sí y más aún se reconozcan unas con otras, como sujetos que están enfrentando una experiencia común. Se apuesta a que, a través del desarrollo de las sesiones grupales, las madres vayan constituyéndose como integrantes de un grupo y que lleguen a visualizar este espacio como una red de apoyo actual y futura (González, 1991).

La modalidad de intervención grupal presenta los siguientes aportes: "representan una fuente de apoyo afectivo, un medio que les permite salir de la rutina, donde desarrollan habilidades de comunicación, adquieren mayor seguridad y soltura para enfrentar su vida y mejorar su autoestima, además de aportar soluciones para problemas específicos, según sus objetivos" (Aylwin y Solar, 2002, p. 186). Además, potencia el intercambio de experiencias entre personas que están en situaciones similares y, con ello, el fomento de las redes de apoyo social informal, tan necesarias siempre para el desempeño de la maternidad y la paternidad y especialmente en situaciones de dificultad proporciona un escenario sociocultural de construcción del conocimiento en el que se potencia el respeto ante la diversidad de puntos de vista y el aprendizaje de comportamientos alternativos (Hidalgo, Menéndez, Sánchez, Lorence y Jiménez, 2009, p. 419).

\section{Reflexiones finales}

Una vez presentado este diseño de intervención es relevante destacar a modo de reflexiones finales, dos aspectos: explicitar el horizonte de cambio al cual apunta esta intervención e identificar de un set de condiciones mínimas que podrían favorecer su implementación en el contexto escolar secundario.

En relación a las apuestas de transformación social a la base del diseño de intervención es posible reconocer: Primero, este diseño de intervención busca construir a la transformación social, reconociendo la importancia del involucramiento familiar colaborativo y desde ahí, orientándose a fortalecer y resignificar el vínculo 
familia-escuela. Este vínculo es crucial como condición que favorece los procesos educativos de niños/as y jóvenes. En este caso el vínculo se expresa en la actoría de las madres en un momento específico de la trayectoria educativa, como lo es la transición a la educación media científico-humanista (Liceos Emblemáticos) en Chile. Segundo, en relación al horizonte de cambio que se proyecta a nivel microsocial en el contexto familiar, este se orienta a potenciar transformaciones que implican el fortalecimiento de los roles y/o las funciones de los diversos actores - especialmente de las madres - quienes mayormente se comprometen en los procesos educativos de los niños/as y jóvenes, posibilitando que ellas transiten de niveles iniciales de participación a niveles superiores y más complejos de participación. En tercer lugar, la intervención también orienta su horizonte de transformación a la familia como tal, ellas pueden resignificar su participación en el ámbito escolar desde una perspectiva de empoderamiento para el ejercicio de sus derechos y no solo desde una mirada meramente normativa del cumplimiento social de una función parental. En cuarto lugar, la intervención tiene un ámbito de transformación que impacta en la escuela, la forma en que se concibe el fenómeno educativo, cada vez más complejo y multidimensional, donde no es posible obviar el rol de la familia en la labor de la escuela, considerándola un aliado estratégico. Toda vez que la escuela comprende este involucramiento parental-materno y lo integra en sus instrumentos de mejora, está avanzando en la implementación de una mejora escolar integradora y contextualizada.

En relación a las condiciones mínimas para que este diseño de intervención se pueda implementar y proyectar en el tiempo, se estima que al menos se deben dar este conjunto de condiciones: Un diseño de esta naturaleza sería esperable que se inscriba en un plan de mejora global de las comunidades educativas, tributando a los lineamientos que apunten a favorecer el involucramiento familiar colaborativo en lógica de mejora continua, es decir, reconociendo la recursividad de los procesos de cambios. En síntesis, la escuela debe dar garantías de voluntad política para su realización y propiciar los recursos y procesos para su implementación en coherencias con sus propias apuestas de cambio. Por ejemplo, podría canalizarse mediante los planes de mejoramiento SEP, podría ser parte de los lineamientos de convivencia escolar o de estrategias que apunten a mejorar el clima escolar (Santana, 2014). Una segunda condición asociada a la perspectiva de la gestión de la escuela y sus opciones técnico-pedagógicas se relaciona a la visión que la escuela tiene de las trayectorias educativas y a los principales hitos de estas, entre ellos, de los procesos de tránsito. Escuelas sensibilizadas con los hitos de transición tendrán una mejor disposición a implementar acciones que los favorezcan y que tengan efectos positivos directos e indirectos. El conocimiento de los perfiles de los estudiantes, las particularidades de sus trayectorias, las características de los tránsitos de ciclos y de establecimientos, permiten que las autoridades, profesores y otros profesionales puedan ir generando mejores condiciones para los aprendizajes de los niños/as y jóvenes. En tercer lugar, una condición mínima es que la escuela provea a los profesionales idóneos para la implementación de esta intervención, es decir, que se cuente con un equipo profesional - idealmente compuestos por trabajadores sociales y otros afines - que posean las competencias para realizar acompañamientos individuales y grupales a las madres, con dominio técnico de las temáticas asociadas a la educación, la participación, la alianza familia- escuela y el reconocimiento de la educación como posibilidad de mejorar las condiciones de vida. Quienes sean capaces de vincularse positivamente con las madres generando relaciones de confianza que enriquezcan cada una de las acciones que se ejecuten y, por ende, se enriquezca el proceso individual de cada una de ellas. Los que adicionalmente puedan articular su quehacer con la gestión de la escuela. En la actualidad, los establecimientos públicos chilenos, en su mayoría han ido incorporando profesionales del área psicosociales que implementan programas específicos, por ejemplo, encargados de convivencia escolar, profesionales SEP para el ámbito psicosocial, entre otros (Saracostti, Santana y Lara, 2019), ellos podrían encargarse de implementaciones de esta naturaleza. En cuarto lugar, el desarrollo de esta intervención debe establecer los puentes con la actual política educativa a nivel nacional, vislumbrando de qué manera los lineamientos y recursos disponibles actualmente en el sector educativo pueden ser aquello que aporte proyección y sustentabilidad a este tipo de apuestas. Si bien, este diseño de intervención puede considerarse para una experiencia piloto, puede tener impactos en el mediano y largo plazo, llevando a las escuelas a problematizar respecto a la relación familia escuela, los tránsitos educativos y las condiciones de aprendizaje. 
Finalmente, otra condición mínima es que las madres (potenciales participantes) tengan un genuino interés por ser parte de estos procesos, respetando sus intereses, voluntad y autodeterminación en la forma en que ellas deseen canalizar el apoyo a sus hijos/as en el ámbito escolar, resultando un imperativo ético contar con su participación voluntaria y con sentido para ellas mismas. Ámbito que se contempla en las etapas iniciales de convocatoria, explorando en sus intereses y voluntad de participar.

\section{Bibliografía}

1. Acuña-Collado, V. (2016). Familia y escuela: crisis de participación en contextos de vulnerabilidad. Revista Brasileira de Estudos Pedagógicos, vol. 97, 246, pp. 255-272.

2. Ames, P. y Rojas, V. (2011). Cambios y oportunidades: La transición de la escuela primaria a la secundaria en el Perú. GRADE, Niños del Milenio, Documento de Trabajo, 63. Lima.

3. Anderson, S. y Carrasco, A. (2013). Nuevas perspectivas para la mejora escolar en tiempos de crecientes políticas de estandarización y rendición de cuentas: evidencia proveniente del congreso ICSEI 2013. Pensamiento educativo. Revista de Investigación Educacional Latinoamericana. 50,2, pp- 1-4.

4. Arancibia, V. (1997). El rol de la madre como mediadora en el proceso de habilitación. Estudios Públicos, 59 (invierno).

5. Aylwin, N. y Solar, M. (2002). Trabajo Social Familiar. Santiago: Ediciones Universidad Católica de Chile.

6. Belfield, C. \& Levin, H. (2002). The effects of competition on educational outcomes: a review of US evidence. Columbia Universito, New York.

7. Bernardi, F. y Cebolla, H. (2014). Clase social de origen y rendimiento escolar como predictores de las trayectorias educativas. Revista Española de Investigaciones Sociológicas, 146, pp. 3-22.

8. Beutel, A., \& Anderson, K. (2008). Race and the educational expectations of parents and children: The case of South Africa. The Sociological Quarterly. Vol. 49, 2, pp. 335-361.

9. Brunner, J., y Elacqua, G. (2004). Factores que inciden en una educación efectiva. Evidencia internacional. Manuscrito. Santiago: Universidad Adolfo Ibáñez.

10. Chacón, E., Chacón, M., Alcedo, Y. y Suárez, M. (2015). Capital cultural, contexto familiar y expectativas en la educación media. Acción Pedagógica, vol. 24, 1, pp. 6-19.

11. Chen, Y. (2007). Principals' Distributed Leadership behaviors and their impact on student achievement in selected elementary school in Texas. Texas: Texas $A \& M$ Univesity.

12. Chinchilla, $\boldsymbol{R}$. (2015). Trabajo con una familia. Un aporte desde la orientación familiar. Revista Electrónica Actualidades Investigativas en Educación, Universidad de Costa Rica, Vol. 15, 1, DOI: dx.doi.org/10.15517/aie.v15i1.17631.

13. Coe, J., Davies, P. \& Sturge-Apple, M. (2017). The multivariate roles of family instability and interparental conflict in predicting children's representations of insecurity in the family system and early school adjustment problems. Journal of Abnormal Child Psycology, 45, 2, pp. 211-224.

14. Cowan, G., Bobby, K., St Roseman, P. \& Echandia, A. (2002). Evaluation Report: The Home visit project. Retrieved from ERIC database.

15. Dabas, E. (2005). Redes sociales, familias y escuela. Buenos Aires: Paidós.

16. Demissie, Z. \& Brener, N. (2017). Mental health and social services in schools: variations by school characteristics - United States, 2014. Mental Health \& Prevention, 5, pp. 5-11.

17. Deslandes, R. (2019). A framework for school-family collaboration integrating some relevant factors and processes. Aula Abierta, vol. 48, 1, pp. 11-18.

18. Duppong, K., Lambert, M., January, S. \& Huscroft, J. (2017). Confirmatory factor analyses comparing parental involvement framewoks with secondary students. Psychol Schs, 54, pp. 947-964.

19. Elmore, R. (2010). Mejorando la escuela desde la sala de clases. Santiago: Fundación Chile.

20. Epstein, J. (2018). School, family, and community partnerships in teachers'profesional work. Journal of Education for Teaching, vol. 44, 3, pp. 397-406.

21. Fernández, C., García, O. y Rodríguez, S. (2016). Los padres y las madres ante la toma de decisiones académicas de los adolescentes en la educación secundaria. Un estudio cualitativo. RMIE, vol. 21, 71, pp. 1111-1133.

22. Ferrara, M. (2015). Parent Involvement Facilitators: Unlocking Social Capital Wealth. School Community Journal. Vol. 25, 1, pp. 29-51.

23. Filardo, V. (2016). Integralidad en el análisis de trayectorias educativas. Educacao \& Realidade, Porto Alegre, vol. 1, 1, pp. 15-40.

24. Goldfarb, S., Locher, J., Preskitt, J., Becker, D., Davies, S. \& Sen, B. (2017). Associations between participation in family activities and adolescent school problems. Child: care, health and development, 43, 3, pp. 361-368.

25. Gomilla, M. y Pascual, B. (2015). La participación de las familias en el sistema educativo: la percepción del profesorado en formación. Revista Electrónica Interuniversitaria de Formación del Profesorado, 18. Recuperado de: http://dx.doi.org/10.6018/reifop.18.3.199321.

26. González, M. (1991). El taller de los talleres. Colección para el nivel terciario. Serie Educación Inicial. Buenos Aires: Estrada.

27. Greenberg, M., Domitrovich, C. \& Brumbarger, B. (2000). Preventing Mental Disorders in School-Age Children: A Review of Prevention Programs. Prevention Research Center for the Promtion of Human Development. Pennsylvania State University. Recuperado en: http://prevention.psu.edu/pubs/documents/ MentalDisordersfullreport.pdf

28. Greene, R. (2008). Human Behavior Theory and Social Work Practice. New Brunswick: Transition Publishers.

29. Grolnick, W. (2015). Mothers'motivation for involvement in their children's schooling: mechanisms and outcomes. Motivation and Emotion, 39 , pp. 63-73.

30. Gubbins, V. (2012). Familia y escuela: tensiones, reflexiones y propuestas. Docencia, $N^{\circ} 46$, pp. 64-73.

31. Gubbins, V. (2016). Relación familias y escuela: ¿Por qué y para qué? Santiago: Ediciones Universidad Finis Terrae.

32. Gubbins, V. e Ibarra, S. (2016). Estrategias educativas familiares en Enseñanza Básica: análisis psicométrico de una escala de prácticas parentales. Psykhe, $25,1, p p .1-17$.

33. Gubbins, V.y Otero, G. (2018). Determinants of parental involvement in primary school: evidence from Chile. Educational Review, 1-20, pp. 9-34.

34. Hidalgo, M., Menéndez, S., Sánchez, J., Lorence, B. y Jiménez, L. (2009). La intervención con familias en situación de riesgo psicosocial. Aportaciones desde un enfoque psicoeducativo. Colegio Oficial de Psicología de Andalucia Occidental, Universidad de Cádiz, Universidad de Huelva y Universidad de Sevilla. Vol. 27, número 2-3. 
35. Imbernon, F. y Medina; J. (2008). Metodología participativa en el aula universitaria. La participación del alumnado. Barcelona: Universitat de Barcelona - Institut de Ciéncies de L'Educación.

36. Jensen, K., Joseng, F. y Lera, M (2007). Familia y Escuela. Recuperado de: http://www.golden5.org/golden5/golden5/programa/es/5FamiliayEscuela.pdf 37. Jiménez, E., Ito, E. y Macotela, S. (2010). El papel de las madres en la motivación de sus hijos (as) hacia el aprendizaje escolar. Revista Mexicana de Psicología Educativa (RMPE), 1, 1, pp. 59-74.

38. Kelly, M., Thompson, A., Frey, A., Klemp, H., Alvarez, M. \& Cosner, S. (2015). The State of School Social Work: Revisited. School Mental Health, 7, 3, pp.174-183.

39. Landaverde, M. (2011). Guía para desarrollar talleres y orientaciones con padres y madres de familia. San Luis de Potosí: Secretaría de Educación de Gobierno de Estado y Sindicato Nacional de trabajadores de la educación.

40. Lareau, A. \& Lopes, V. (2012). You're not going to call the shots. Sociology of Education. Vol. 85, 3, 201-218.

41. Lareau, A. (2015). Cultural knowledge and social inequality. American Sociological Review. Vol. 80, 1, pp. 1- 27.

42. Levin, J. y Datnow, A. (2012). The Principal Role in Data Driven Decision Making: using case study to developmulti-mediator moldels of educational reform. School effectiveness and School Improvement, 23, 2, pp.179-201.

43. Ley $N^{\circ}$ 20.248. Diario Oficial de la República de Chile, Santiago, Chile, 25 de enero de 2008.

44. Ley $N^{o}$ 20. 370. Diario Oficial de la República de Chile, Santiago, Chile, 28 de julio de 2009.

45. Liang, $\boldsymbol{Y}$. \& Zhang, S. (2016). Construction of a service mode of school social work in post-disaster areas in China: A case study on the project of disaster relief schools after the Sichuan earthquake. International Social Work. Vol.59, 6, pp.760-777.

46. Manríquez, B. (2016). Efectos le los Liceos Públicos Emblemáticos sobre el desempeño escolar: El Caso del Liceo $N^{\circ} 1$ de Niñas de Santiago Javiera Carrera. Postgrado Economía y Negocios, Universidad de Chile, Santiago.

47. Maureira, F. (2016). Con el reto de mejorar simultáneamente la calidad y la equidad Chile transforma su sistema escolar. Tarea, 93, pp. 35-39.

48. Mejías, E. (2014). Participación de madres de sectores vulnerables en colegios de excelencia académica (Actividad de Graduación de Magister), Pontificia Universidad Católica de Chile, Santiago, Chile.

49. Mejías, E., Santana, A., Saracostti, M., Reininger, T. y Lara, L. (2019). Involucramiento parental-materno. Trayectorias vitales maternas y procesos de participación escolar en la transición a la Educación Media. En: La relación entre familias y escuelas en Chile. Aprendizajes desde la política educativa, la investigación y la intervención socioeducativa. M. Saracostti, A. Santana, L. Lara.(Eds.). Santiago: RIL Editores.

50. Mercado, R. y Montaño, L. (2015). Procesos de participación entre profesoras de jardines de niños y madres de familia en actividades de enseñanza. RMIE, vol. 20,65, pp. $347-368$.

51. MINEDUC. (2017). Politica de participación de las familias y la comunidad en instituciones educativas. Santiago: División Educación General.

56. Montiel-Castillo, V.y Guerra-Morales, V. (2016). La psicoeducación como alternativa para la atención psicológica a las sobrevivientes de cáncer de mama. Revista Cubana de Salud Pública, vol. 42, 2, pp. 332-336.

57. Muñoz, G., Marfán, J., Pascual, J., Sánchez, M., Torre, M. y Von Hausen, C. (2010). Planes de Mejoramiento SEP: sistematización, análisis y aprendizaje de politica. Santiago: Fundación Chile-Mide UC-CEPPE.

58. Ospina-Botero, M. (2016). Tejiendo redes: escuela y familia. Revista Inclusión \& Desarrollo, 3 (I), pp. 80-91.

59. Park, S. \& Holloway, S. (2017). The effects of school-based parental involvement on academic achievement at the child and elementary school level: a longitudinal study. The Journal od Educational Research, vol. 110, 1, pp. 1-16.

60. Pérez, J., Alvarez, L. y Cubillos, M. (2016). Relación entre nivel académico y metas de socialización en madres de niños preescolares. Diversitas, vol. 12, 1, pp. 13-25.

61. Reininger, T. y Santana, A. (2017). Parental involvement in municipal schools in Chile: Why do parents choose to get involved? School Psychology International. Vol. 38, 4, pp. 1-17.

62. Reinke, W., Smith, T. \& Herman, K. (2019). Family-School Engagement Across Child and Adolescent Development. School Psychology, vol. 34, 4, pp. 346-349.

63. Romagnoli, C. y Cortese, I. (2015). ¿Cómo la familia influye en el aprendizaje y rendimiento escolar?. Ficha VALORAS actualizada de la $1^{\circ}$ edición "Factores de la familia que afectan los rendimientos académicos" (2007). Disponible en Centro de recursos VALORAS: [www.valoras.uc.cl].

64. Roy, M. \& Giraldo-García, R. (2018). The roles of parental involvement and social/emotional skills in academic achievement: global perspectives. School Community Journal, vol. 28, 2, pp. 29-46.

65. Sánchez, A., Reyes, F. y Villarroel, V. (2016). Participación y expectativas de los padres sobre la educación de sus hijos en una escuela pública. Estudios Pedagógicos, XLII, 3, 347-367.

66. Santana, $\boldsymbol{A},\left(2010^{\circ}\right)$. Cultura juvenil y cultura escolar. Aproximación a una relación compleja: Tensiones y Desafios. Foro Educacional, 17, pp.45-63.

67. Santana, A. (2010b). Relación Familia-Escuela en contextos de pobreza. Posibilidades y limitaciones en los procesos educativos. CUHSO Cultura, Hombre y Sociedad, vol. 20, 2, pp.81-93

68. Santana, A. (2014). Social Intervention in Early Education: Initial strategies for dealing with education in situations of extreme poverty as a function of the Family-School relationship: The case of Chile. Procedia Social and Behavioral Sciences. 132, pp. 285-290.

69. Santana, A. y Reininger, T. (2017). El involucramiento familiar en el marco de la relación Familia-Escuela. Un análisis desde el Equipo de Gestión Escolar en una Escuela Municipal chilena. Comunitania. Revista Internacional de Trabajo Social y Ciencias Sociales. 4, pp. 55-74.

70. Saracostti, M., Santana, A. y Lara, L. (2019). La relación entre familias y escuela en Chile. Aprendizaje desde la política educativa, la investigación y la intervención socioeducativa. Santiago: RIL Editores.

71. Taylor, S. y Bogdan, R. (2002). Introducción a los métodos cualitativos de investigación. Barcelona: Paidós Básica.

72. Vásquez, N., Zuluaga, N. y Fernández, D. (2017). Clima escolar y funcionalidad familiar como factores asociados a la intimidación escolar en Antioquia Colombia. Pensamiento Psicológico, 15, 1, 63-72.

73. Vélaz, C., López, E., Expósito, E. y González, A. (2016). El enfoque intersectorial en la provisión de orientación y apoyo escolar. Perspectiva de orientadores, tutores y directores. Revista Complutense de Educación, Vol. 27, 3, pp. 1271-1290.

74. Yi Hung, E. \& Power, T. (2018). Parental involvement during the transition ti primary school: examining bidirectional relations with school adjustment. Children and Youth Services Review, vol. 88, pp. 257-266. 